\title{
Análise dos indicadores de intensidade de P\&D: entendendo os efeitos da estrutura industrial e dos gastos setoriais
}

Analysis of indicators of intensity of P\&D: understanding the effects of the industrial structure and sectoral expenditures

\author{
Paulo César Brigante (1) \\ (1) Universidade Estadual Paulista
}

\begin{abstract}
This article examines the determinants of $R \& D$ business expenditures through an $\mathrm{R} \& \mathrm{D}$ intensity indicator defined by the ratio between R\&D expenditures and industry value added. With the decomposition of its determinants, the objective of this article is to identify the extent to which Brazil's distance in terms of technological efforts, measured by such indicator, in relation to the average of a set of selected countries, can be attributed to the effects of the industrial structure R\&D expenditures made by the different technology groups that make up this industry.
\end{abstract}

Resumo

Este artigo procura analisar os determinantes de

\section{Keywords} gastos empresariais em P\&D por meio de um indicador de intensidade definido pela relação entre gastos em P\&D e valor adicionado da indústria. Com a decomposição de seus determinantes, o objetivo é identificar em que medida a distância do Brasil em termos de esforços tecnológicos, medidos por tal indicador, em relação à média de um conjunto de países selecionados, pode ser atribuída aos efeitos da estrutura industrial predominante $e$ aos gastos em PQD efetuados pelos diferentes grupos tecnológicos que compõem esta indústria.

Intensity R \& D; Innovation Policies; Business Expenses in R\&D.

JEL Codes O3.

\section{Palavras-chave}

Intensidade de P\&D; Políticas de Inovação; Gastos Empresariais em P\&D.

Códigos JEL O3. 


\section{Introdução}

Nas abordagens que tratam da natureza das variações dos esforços tecnológicos e do seu significado econômico, as estruturas industriais de diferentes países ocupam papel relevante. A questão dos esforços tecnológicos da indústria é analisada sob uma ênfase comparativa em que países mais desenvolvidos exibem níveis mais elevados que os menos desenvolvidos.

Em tais análises, os gastos em pesquisa e desenvolvimento de um país (P\&D) em relação a alguma variável agregada da atividade econômica como produto interno bruto, vendas líquidas ou valor adicionado - é o indicador comumente utilizado. A denominação recebida por este indicador é a de intensidade de $\mathrm{P} \& \mathrm{D}$, para a qual ocorre variação de valores entre os países. Enquanto que para alguns ele pode ser elevado, caracterizando níveis altos de esforços tecnológicos, para outros ele é baixo. Essa variação leva ao estabelecimento de um ranking de posições em que um determinado país pode ser comparado em relação àqueles com melhores indicadores, por exemplo.

As análises comparativas enquadram-se no debate sobre políticas industriais e de inovação por trazerem implicações quanto ao desenho e instrumentos mais eficazes para reduzir as defasagens tecnológicas entre os países. Nesse sentido é que alguns trabalhos consideraram a importância, em nível setorial, de maiores montantes de gastos que as empresas destinam à $\mathrm{P} \& \mathrm{D}$ em relação ao valor adicionado. Da mesma forma, também mostraram o papel exercido pela estrutura industrial e concluíram que ambos produzem reflexos sobre os gastos totais em $\mathrm{P} \& \mathrm{D}$ em um país.

$O$ presente artigo tem como objetivo identificar em que medida a distância do Brasil em relação a outros países selecionados atribui-se aos componentes: estrutura industrial e esforços tecnológicos. Estes últimos como sendo a intensidade de $\mathrm{P} \& \mathrm{D}$ dos grupos tecnológicos que compõem a indústria de transformação e seguindo a classificação da Organização para o Comércio de Desenvolvimento (OCDE). Por tal critério, os setores são agregados em quatro grupos, conforme a intensidade tecnológica média de cada um, dividindo-se em: alta tecnologia, média-alta, média-baixa e baixa tecnologia.

Com o auxílio dos indicadores de intensidade de $\mathrm{P} \& \mathrm{D}$ para cada país da amostra, aplicou-se uma metodologia de decomposição quantitativa com 
base em Sandven e Smith (1998) ainda não utilizada em outros trabalhos da área no Brasil. Com ela foi possível distinguir o quanto cada grupo tecnológico contribui em termos de gastos em $\mathrm{P} \& \mathrm{D}$ para a média da indústria e, também, em termos de participação no valor adicionado. Com a extensão desse critério para um conjunto mais amplo de países, que serviu como base de comparação para o Brasil, foi possível identificar o quanto das nossas diferenças pode ser atribuída a cada um daqueles componentes mencionados acima.

Dessa forma espera-se contribuir para o debate sobre políticas industriais e de inovação ao se demonstrar a possibilidade deste outro enfoque comparativo. Ao mesmo tempo que permite um melhor entendimento sobre a importância do indicador de intensidade de P\&D e seu papel.

A aplicação dessa metodologia foi possível devido à disponibilidade de dados referentes ao valor adicionado das empresas inovadoras no Brasil (VTI), bem como de seus gastos em P\&D. Isso permitiu que a construção dos indicadores fosse semelhante à utilizada para a maioria das análises que utilizam dados da OCDE, já que alguns trabalhos no Brasil utilizam a receita líquida de vendas enquanto denominador no indicador.

Vale lembrar que, no Brasil, nos anos 2000, com a retomada do crescimento econômico esperava-se que as empresas acentuassem o ritmo de inovações. Naquele período, associaram-se várias modificações no aparato institucional da inovação, além de ações implementadas no âmbito de políticas industriais e tecnológicas. Entre outras destacaram-se: a Política Industrial, Tecnológica e de Comércio Exterior (PITCE), de 2004, a Política de Desenvolvimento Produtivo (PDP), de 2008 e o Plano Brasil Maior (PBM), de 2011.

Diante daquele contexto, reforçou-se a necessidade de uma melhor compreensão a respeito do comportamento empresarial em termos de novos investimentos em $\mathrm{P} \& \mathrm{D}$, já que aquelas iniciativas contemplavam, explicitamente, a necessidade do aumento dos esforços tecnológicos empresariais. Apesar disso, conforme será visto adiante, os indicadores não demonstraram evoluções significativas apontando uma direção persistente de redução da defasagem tecnológica do Brasil em relação às maiores economias do mundo.

Além desta introdução, o artigo está organizado da seguinte forma: a próxima seção trata dos gastos em $\mathrm{P} \& \mathrm{D}$, destacando sua importância principalmente para a literatura empírica, assim como para sua aplicação 
segundo níveis de agregação de atividades. Destacam-se, nestes últimos, os gastos empresariais em $\mathrm{P} \& \mathrm{D}$ realizados na indústria de transformação e sua relação com o valor adicionado total da indústria. Nas abordagens tradicionais, o indicador de intensidade é uma referência para comparações de desempenho inovativo entre os países e, recentemente, tem sido utilizado para a definição de metas de políticas industriais e tecnológicas. As limitações quanto ao uso, bem como a importância da estrutura industrial e dos gastos setoriais tratados pela literatura recente também são abordados nesta seção.

Uma breve discussão em relação ao papel da estrutura industrial e dos gastos setoriais enquanto determinantes da intensidade de P\&D e aos objetivos de política industrial e de inovação é feita no subitem 2.2.

$\mathrm{Na}$ seção 3 detalha-se a metodologia aplicada para avaliar os efeitos de cada um dos determinantes sobre os gastos empresariais em P\&D. Foram utilizados dados de gastos em P\&D, interna e externa, da Pesquisa de Inovação (Pintec) e, também, da Pesquisa Industrial Anual (PIA), ambas do Instituto Brasileiro de Geografia e Estatística (IBGE). A análise foi empregada para os anos de 2000, 2005 e 2011 e, por meio da classificação em grupos tecnológicos da OCDE, tornou possível uma melhor compreensão daqueles efeitos em cada grupo tecnológico específico.

Após a formalização de como a metodologia pode ser aplicada para a indústria de transformação de um único país, demonstra-se como é possível a mesma aplicação para um conjunto de países. Obtém-se, assim, uma medida dos impactos exercidos pela estrutura industrial sobre as intensidades totais de $\mathrm{P} \& \mathrm{D}$ da indústria e, ao mesmo tempo, pelas intensidades de $\mathrm{P} \& \mathrm{D}$ dos grupos.

$\mathrm{Na}$ seção 4, a partir dos resultados da seção 3, realizam-se algumas comparações do Brasil com um conjunto de países selecionados a fim de verificar em que medida efeitos gerados pela estrutura industrial e pelas intensidades respondem por uma maior distância e/ou aproximação em relação àquele conjunto.

Conclui-se discutindo algumas implicações em termos de políticas e ressaltando a necessidade de se compreender como as mesmas atuam sobre a estrutura industrial e sobre os gastos em $\mathrm{P} \& \mathrm{D}$, bem como da interdependência entre estes. Aspecto que seria fundamental para o desenho, elaboração e resultados mais eficazes das políticas industriais e de incentivo à inovação e $P \& D$ no Brasil. 


\section{Revisão da literatura}

\subsection{Aspectos relativos ao uso do indicador de intensidade de P\&D}

Há uma vasta literatura empírica que trata da importância dos gastos em pesquisa e desenvolvimento $(\mathrm{P} \& \mathrm{D})$ e sua correlação com o crescimento econômico na qual eles são definidos como insumos que contribuem para o processo de produção (Griliches, 1998). Em sua maioria, tais estudos têm concluído pela existência de uma associação positiva deles com o crescimento em longo prazo de um determinado país (Pavitt K.; Patel P., 1994).

Os gastos em $\mathrm{P} \& \mathrm{D}$ são utilizados na construção de indicadores de ciência e tecnologia para vários países e, como tais,são relevantes instrumentos para as decisões de políticas. Entre esses indicadores, o mais utilizado é o de intensidade de $\mathrm{P} \& \mathrm{D}$, que reflete a capacidade inovadora de um país e pode ser calculado conforme alguns níveis de agregação.

$O$ indicador de intensidade, quando se refere à economia nacional, é definido pela relação entre os gastos totais em $\mathrm{P} \& \mathrm{D}$ e o produto interno bruto (PIB). Nesse caso são considerados todos os gastos domésticos realizados em $\mathrm{P} \& \mathrm{D}$ pelo setor empresarial (público e privado), além daqueles realizados pelo governo, pelas universidades e instituições de pesquisa e, também, pelas instituições sem fins lucrativos. No entanto, é possível calculá-lo somente para a indústria de transformação, na qual os gastos empresariais realizados neste setor são divididos pelo valor adicionado total, ${ }^{1}$ (Galindo-Rueda, F.; Verger, F., 2016).

Suas limitações são bem conhecidas na literatura, sobretudo por ser um indicador de esforços ${ }^{2}$ e não de resultados em termos de produção cien-

1 Os cálculos deste último indicador, para o caso brasileiro, implicam certos cuidados metodológicos, conforme apontam Araújo e Cavalcante (2011). Pois é preciso considerar que a intensidade de $\mathrm{P} \& \mathrm{D}$ pode ser expressa por alguns indicadores como, por exemplo, $\mathrm{P} \& \mathrm{D} /$ RLV (gastos em P\&D sobre a receita líquida de vendas) e/ou P\&D/PIB que, no entanto, não são idênticos.

Em um caso, o quociente é, essencialmente, o faturamento, e, no outro, o quociente é uma proxy do valor agregado. Além disso, para efeito de comparações internacionais, como será o caso aqui, os dados referentes aos gastos em P\&D deveriam ser obtidos de forma metodologicamente consistente com o faturamento, ou o valor agregado, referindo-se ao mesmo plano amostral. No Brasil, o problema é que os gastos em $\mathrm{P} \& \mathrm{D}$ geralmente são obtidos nas pesquisas de inovação, a Pintec, e o valor adicionado, nas contas nacionais. As tabulações especiais do IBGE, nas quais se disponibilizaram os valores da transformação industrial (VTIs) somente das empresas inovadoras, buscaram contornar tais limitações metodológicas tornando a análise mais consistente.

2 No entanto, de acordo com Cohen e Levinthal (1989), o aumento das atividades de P\&D 
tífica e tecnológica. Entretanto, seu uso é difundido em termos mundiais, uma vez que vem sendoaplicado há aproximadamente quarenta anos nos países da OCDE.

Enquanto uma referência às decisões de política, essa aplicação estende-se às diversas análises comparativas internacionais sobre performances de inovação. ${ }^{3}$ Esse último aspecto implica alguns questionamentos sobre o indicador, entre eles, quais seriam os fatores que explicam o seu aumento ao longo do tempo. E, apesar da ampla utilização e importância, são escassos os estudos voltados ao entendimento da estrutura industrial e dos gastos setoriais enquanto determinantes do mesmo.

A necessária compreensão se torna evidente visto que algumas análises recentes demonstraram que a tentativa de aumentar gastos em $\mathrm{P} \& \mathrm{D}$ das empresas podenão resultar em aumentos diretos na intensidade total do país, (Kayal, 2015). Pois a intensidade total pode ser um forte reflexo de sua estrutura industrial onde estaria presente um maior peso dos setores mais intensivos em $\mathrm{P} \& \mathrm{D}$ em relação ao seu produto.

Tal aspecto já havia sido tratado por Sandven e Smith (1988), que sistematizaram as relações entre estrutura industrial e intensidade de $\mathrm{P} \& \mathrm{D}$ e avaliaram os efeitos sobre a intensidade total de $\mathrm{P} \& \mathrm{D}$ da indústria para vários países da OCDE. A conclusão foi que a maior parte das diferenças observadas nas intensidades de $\mathrm{P} \& \mathrm{D}$ entre as economias resultavam da estrutura industrial que poderia ser mais ou menos voltada aos segmentos de maior intensidade tecnológica.

Alguns poucos estudos comparativos que buscaram identificar a influência de outros fatores sobre a intensidade de $\mathrm{P} \& \mathrm{D}$ de um determinado país concluíram pela maior influência da estrutura industrial, (Mathieu; La Potterie, 2008). Esse estudo realizou uma análise econométrica com dados em painel de 18 países entre 2001 e 2005 e a estrutura industrial foi representada por dummies para 21 setores. Entre os outros fatores es-

contribuem também para o aumento da capacidade de absorção e aprendizado das empresas, tornando possível melhorar a assimilação, exploração e aprimoramento de conhecimentos já existentes.

3 As estatísticas referentes à $\mathrm{P} \& D$ são provavelmente as mais importantes entre os diferentes tipos de indicadores da atividade inovativa. Sua metodologia incorpora as propostas da Organização para a Cooperação e Desenvolvimento Econômico (OCDE) contidas no Manual de Frascati. Este acabou se tornando uma referência de normas internacionais e padronizadas para a produção e metodologia aplicada aos surveys. Atualmente, as mesmas são aplicadas em grande parte dos países em desenvolvimento, embora seu enfoque original tenha sido escrito para a elaboração dos surveys de P\&D nos países da OCDE (OECD, 2015)(MONTMARTIN, 2012). 
colhidos estão os sistemas nacionais de inovação, as políticas científicas e tecnológicas e o tamanho do mercado.

A hipótese de que a estrutura industrial importa na composição da intensidade de P\&D também foi corroborada por Kayal (2015). O autor calculou a intensidade de $\mathrm{P} \& \mathrm{D}$ para cada setor da indústria de transformação e agrupou os países conforme suas respectivas intensidades de $\mathrm{P} \& \mathrm{D}$. Com isso atribui um critério de classificação em que as mesmas se subdividiam em alta ou baixa em relação a uma média calculada entre os países. Para cada setor, realizou-se um teste de diferença de médias entre o valor adicionado dos países com alta e os de baixa intensidade de $\mathrm{P} \& \mathrm{D}$, confirmando que a diferença era significativa (Kayal, 2015).

No Brasil, em geral, são escassos os estudos que buscam entender os indicadores relativamente limitados de esforços tecnológicos na indústria de transformação e que baseiam-se emcomparações com um número razoável de países.

Zuccoloto e Toneto Jr. (2005), por exemplo, buscaram observar se o baixo esforço inovador da indústria brasileira decorre dos insuficientes esforços tecnológicos, representados por indicadores setoriais de $\mathrm{P} \& \mathrm{D}$, da discrepância entre o esforço nacional e estrangeiro em um determinado grupo de indústrias, e/ou da diferença entre a estrutura produtiva brasileira. Em uma amostra de 19 países para o ano de 2000, concluíram que o limitado esforço dos setores da indústria, sobretudo daqueles intensivos em tecnologia, importa mais do que a estrutura produtiva nacional.

Recentemente outros trabalhos simularam os impactos sobre o indicador de intensidade para os anos 2006 e 2008 por meio da construção de cenários hipotéticos. Neles foram utilizados dados dos EUA, Alemanha e Canadá com suas respectivas estruturas industriais e gastos setoriais (Cavalcante, 2013).

Conforme já havia sido demonstrado em estudo anterior, no Brasil, a participação dos setores menos intensivos em tecnologia é maior na estrutura industrial, implicando em baixa contribuição dos mesmos para os gastos totais em P\&D da indústria (De Negri; Cavalcante, 2013). Por outro lado, os setores mais intensivos, pertencentes aos grupos de alta e média-alta tecnologia, apresentam contribuições mais elevadas para os gastos totais em $\mathrm{P} \& \mathrm{D}$. No entanto, a reduzida participação deste primeiro grupona estrutura industrial resulta em baixa intensidade total de $\mathrm{P} \& \mathrm{D}$ da indústria. 
As tentativas de se encontrar explicações para os insuficientes indicadores de P\&D no Brasil teriam levado a conclusões que a estrutura industrial brasileira é responsável apenas em parte pelo desempenho inferior do país. Isso se deve ao menor peso de setores intensivos em tecnologia, como o eletrônico e farmacêutico, quando comparados a outros casos, como o da Coreia do Sul, por exemplo. Entretanto, a maior parte da diferença entre o Brasil e os demais países resultaria do menor gasto setor a setor e não seria uma consequência apenas da estrutura (Pacheco, 2009).

\subsection{Objetivos de políticas industriais e de inovação}

Não obstante as limitações apontadas antes, recentemente o indicador de intensidade tem sido utilizado também na formulação de metas explícitas de políticas industriais e de inovação que devem ser atingidas em um determinado período de tempo, (Sheehan, J; Wyckoff, A., 2003).

De fato, tal orientação adquiriu maior importância nos países da União Europeia a partir de 2000, com a Agenda de Lisboa e, posteriormente, com o Conselho de Barcelona, nos quais tornavam-se claras as preocupações com a questão da competitividade e do atraso dos países europeus em relação aos EUA e Japão.

A reação em termos de políticas implicou em dar prioridade à elevação dos gastos em $\mathrm{P} \& \mathrm{D}$ e, por sua vez, tal postura reconhecia que o alcance de metas para a intensidade total de $\mathrm{P} \& \mathrm{D}$ em um país dependeria da elevação desta intensidade na indústria de transformação. Isso porque a intensidade de P\&D de um país é, também, o reflexo de sua estrutura industrial.

Supostamente, as políticas industriais e de inovação orientam-se buscando dois importantes objetivos, além de outros: aumentar o volume de gastos em P\&D em relação ao valor adicionado em cada setor da indústria e, também, promover um movimento da economia em direção aos setores mais intensivos em $\mathrm{P} \& \mathrm{D}$. O primeiro relaciona-se diretamente ao componente de gastos setoriais enquanto que o segundo está ligado ao componente da estrutura industrial.

Para algumas abordagens, as contribuições mais elevadas dos grupos de alta emédia-alta tecnologia, mencionadas acima, sugerem que o foco das políticas deveria se restringir a tais grupos. Conforme já dito, apesar do reduzido peso do primeiro, esse maior foco levaria a um aumento dos 
gastos totais em $\mathrm{P} \& \mathrm{D}$ em relação ao PIB do país. Ao mesmo tempo em que as políticas poderiam atuar tanto pelo lado da estrutura quanto pelo lado dos gastos de cada grupo. (De Negri; Cavalcante, 2013).

No caso dos gastos, diversos instrumentos de apoio público foram utilizados nos anos 2000: crédito subsidiado à $\mathrm{P} \& \mathrm{D}$, financiamento em parcerias com universidades e institutos de pesquisa, subvenções, incentivos fiscais, compras públicas e infraestrutura de pesquisa como laboratórios ou universidades. ${ }^{4}$ Já em relação à estrutura, exigir-se-ia das políticas industriais um papel efetivo em preservar, e/ou aumentar, a participação dos setores mais intensivos em $\mathrm{P} \& \mathrm{D}$ no valor adicionado total da indústria.

Portanto, a relevância em termos de políticas ao se estabelecer os determinantes da intensidade de $\mathrm{P} \& \mathrm{D}$, da forma como foi proposta, consiste em compreender a extensão em que cada um dos componentes exerce seus efeitos sobre os gastos agregados em $\mathrm{P} \& \mathrm{D}$. Assim, identificar o potencial dos instrumentos de apoio público à $\mathrm{P} \& \mathrm{D}$, bem como daqueles de política industrial com impactos mais diretos na estrutura produtiva, seria uma possibilidade resultante dessa melhor compreensão.

\section{Metodologia para avaliação dos efeitos dos compo- nentes dos gastos agregados em P\&D}

A literatura aponta que para uma análise em nível setorial, como a proposta neste artigo, em geral constrói-se o indicador dividindo-se os gastos em P\&D pelo valor adicionado, (Galindo-Rueda, F.; Verger F., 2016).

Assim, para os anos de 2000, 2005 e 2011, considerou-se o montante dispendido em $\mathrm{P} \& \mathrm{D}$, interna e externa, pelas empresas em cada setor da indústria de transformação independente de sua fonte de financiamento. ${ }^{5}$ A fonte pode ser própria, pública ou de terceiros. Tais dados referem-se às empresas que implementaram inovações de produto e/ou processos a

4 Os vínculos ocorrem tanto em termos de pesquisa acadêmica quanto de desenvolvimento de produtos. Neste último, por meio de transferência do conhecimento das universidades para empresas.

5 Este critério foi escolhido porque permite comparações mais adequadas com os outros países que constam no banco de dados Analytical Business Enterprise Research and Development (ANBERD) e STAN (Structural Analysis Database International Standard Industry Classification, Rev.3) da Organização para o Comércio e Desenvolvimento (OCDE). Tal base utiliza as intensidades setoriais conforme o critério descrito acima. 
partir da Pesquisa de Inovação (Pintec) realizada pelo Instituto Brasileiro de Geografia e Estatística (IBGE). Ao mesmo tempo, osmesmos foram cruzados com os valores da transformação industrial (VTI), da Pesquisa Industrial Anual (PIA), também do IBGE e consolidados em tabulações especiais baseados na Classificação Nacional de Atividades (CNAE) 1.0. ${ }^{6}$

Pela classificação da OCDE, a seguir, os quatro grupos tecnológicos são compostos pelos respectivos setores de acordo com suas intensidades de $\mathrm{P} \& \mathrm{D}$, não obstante há algumas limitações quanto à possibilidade de desagregações. ${ }^{7}$ Alta intensidade tecnológica: aeroespacial, farmacêutico, informática, eletrônica, comunicações e equipamentos de instrumentação; Média-alta intensidade tecnológica: material elétrico, veículos automotores, química (exclusive o setor farmacêutico), equipamentos de transporte, máquinas e equipamentos; Média-baixa intensidade tecnológica: construção naval, borracha e produtos plásticos, coque, produtos refinados de petróleo, combustíveis nucleares, outros produtos não metálicos, metalurgia básica e produtos metálicos; e Baixa intensidade tecnológica: outros setores e de reciclagem, madeira, papel e celulose, editorial e gráfica, alimentos, bebidas, fumo, têxtil e de confecções, couro e calçados.

No modelo proposto por Sandven e Smith, os gastos totais em $\mathrm{P} \& \mathrm{D}$ $\left(P D_{T}\right)$ de um país podem ser expressos como:

$$
P D_{T}=P D_{1}+P D_{2}+P D_{3}+P D_{4}
$$

Em que $P D_{1}, P D_{2}$, etc... representam o total de gastos em cada um dos quatro grupos tecnológicos da indústria de transformação. ${ }^{8}$

6 A partir de 2008 passou a ser adotada a CNAE 2.0. Até então, utilizava-se a CNAE 1.0, na qual se baseiam os dados de 2000 e 2005 deste trabalho. Na CNAE 2.0 houve algumas mudanças nas agregações entre grupos (3 dígitos) e divisões 2 (dígitos). Assim, para o ano de 2011 foram feitas as adequações necessárias entre grupos e divisões a fim de que respeitassem o mesmo critério adotado pela CNAE 1.0. Para maiores informações sobre as modificações ver: www.concla.ibge.gov.br.

7 É o caso, por exemplo, do setor aeroespacial e da construção naval. Tais itens estão agregados no setor "Outros equipamentos de transporte" e, conforme advertem De Negri e Cavalcante (2013), este setor engloba diferentes categorias de intensidade tecnológica. Assim, a fabricação de aeronaves é classificada como de alta intensidade, a fabricação de trens e o setor naval são de média-alta, e a fabricação de bicicletas, de média-baixa. Dada a impossibilidade de desagregação, a exemplo de outros trabalhos, optou-se por classificá-lo como de média-alta intensidade tecnológica.

8 Alta, média-alta, média-baixa e baixa tecnologia. Lembrando que o total de gastos de cada grupo resulta da soma dos gastos advindos dos seus respectivos setores, de acordo com a classificação da OCDE. 
Sendo a intensidade total de P\&D na indústria, $I_{t}=P D_{t} / V a_{t}$ e $V a_{t}$, o valor adicionado total da indústria, então, dividindo a equação (1) por $V a_{t}$, os esforços tecnológicos totais podem ser expressos como:

$$
I_{t}=P D_{t} / V a_{t}+P D_{1} / V a_{t}+P D_{2} / V a_{t}+P D_{3} / V a_{t}+P D_{4} / V a_{t}
$$

Esta expressão pode ser transformada pela multiplicação de cada um dos componentes por $V a_{i} / V a_{i}$, ou seja 1, e pela substituição do denominador $V a_{t}$ por $V a_{i}$ onde é o valor adicionado de cada grupo $i$ :

$$
I_{t}=\left[\left(P D_{1} / V a_{1}\right)\left(V a_{1} / V a_{t}\right)+\cdots+\left[\left(P D_{4} / V a_{4}\right)\left(V a_{4} / V a_{t}\right)\right]\right.
$$

Como a somatória dos gastos nos setores é dada em 2 por $I_{t}=$ $=\sum_{i}^{n}\left(P D_{i} / V a_{t}\right)$ então, com base em (3) resulta que $I_{t}=\sum_{i}^{n}\left(P D_{i} / V a_{i}\right)$ $\left(V a_{i} / V a_{t}\right)$ em que $i$ representa o grupo tecnológico enquanto a primeira expressão $\left(P D_{i} / V a_{t}\right)$ dá a intensidade de $\mathrm{P} \& \mathrm{D}$ no grupo $i$ e a segunda expressão, $\left(V a_{i} / V a_{t}\right)$ a participação do valor agregado do grupo $i$ no valor agregado total da indústria. Esta última, portanto, são os pesos relativos de cada grupo e podem ser denotados por $w_{i}$. De tal forma que a expressão acima seja:

$$
I_{t}=\sum_{i}^{n}\left(P D_{i} / V a_{t}\right) w_{i}
$$

Assim, pode-se dizer que a intensidade total de $\mathrm{P} \& \mathrm{D}$ na indústria é uma soma das intensidades de $\mathrm{P} \& \mathrm{D}$ dos grupos, as quais são ponderadas pela participação do respectivo valor adicionado em relação ao valor adicionado total, ou seja, pelo peso atribuído a cada grupo tecnológico que pode ser interpretada como a somatória das contribuições que cada grupo dá para a formação da intensidade total da indústria $I_{t}$.

Efetuando esses cálculos para um conjunto de países, é possível obter uma intensidade média a qual servirá para calcular a distância de um determinado país em relação a este conjunto mais amplo.

Para aquele conjunto, cada grupo tecnológico $i$ tem sua própria intensidade de $\mathrm{P} \& \mathrm{D}, I_{i}$. Toma-se a média delas entre todos os países e em cada $i$ define-se uma intensidade média $\bar{I}_{i}$. Com essa obtenção pode-se transformar a expressão (4) em: $I_{t}=\sum_{i=1}^{n}\left(I_{i}+\bar{I}_{i}-\bar{I}_{i}\right) w_{i}$, em que $I_{i}=P D_{i} / V a_{i}$ que resulta em: 


$$
\begin{aligned}
& I_{t}=\sum_{i=1}^{n}\left[\left(\bar{I}_{i} W_{i}\right)+\left(I_{i}-\bar{I}_{i}\right) w_{i}\right] \\
& I_{t}=\sum_{i=1}^{n}\left(\bar{I}_{i} W_{i}\right)+\sum_{i}^{n}\left(I_{i}-\bar{I}_{i}\right) w_{i}
\end{aligned}
$$

A intensidade total de P\&D fica então subdividida em dois componentes distintos. $O$ primeiro deles é: $\sum_{i}^{n}\left(\bar{I}_{i} w_{i}\right)$, a soma das intensidades médias de $\mathrm{P} \& \mathrm{D}^{9}$ para todos os países multiplicada pela participação de cada grupo em relação ao valor adicionado total em cada país. Ele indica quais seriam as diferenças nas intensidades totais entre os países se todos tivessem a mesma intensidade média e, dessa forma, representa o impacto exercido pela estrutura industrial sobre as intensidades totais de $\mathrm{P} \& \mathrm{D}$ da indústria.

O segundo componente, ) $\sum_{i}^{n}\left(I_{i}-\bar{I}_{i}\right) w_{i}$ mede o efeito puro das intensidades de $\mathrm{P} \& \mathrm{D}$ sobre a intensidade total da indústria quando as diferenças na estrutura industrial são consideradas. Assim, para cada país, ele dá a soma das diferenças entre a intensidade de $\mathrm{P} \& \mathrm{D}$ e a intensidade média em cada grupo, com os pesos aí atribuídos sendo definidos como a participação de cada grupo no valor adicionado total da indústria.

Definindo-se $\bar{I}_{t}$ como a média das intensidades totais da indústria entre todos os países, de modo simétrico os dois componentes em (6) podem ser expressos em termos de diferenças de uma média:

$$
I_{t}-\bar{I}_{t}=\sum_{i}^{n}\left(\bar{I}_{t} w_{i}\right)+\sum_{i}^{n}\left(I_{i}-\bar{I}_{i}\right) w_{i}-\bar{I}_{t}
$$

Rearranjando, temos:

$$
I_{t}-\bar{I}_{t}=\sum_{i}^{n}\left[\left(\bar{I}_{i} w_{i}\right)-\bar{I}_{t}\right]+\sum_{i}^{n}\left(I_{i}-\bar{I}_{i}\right) w_{i}
$$

Para a indústria de transformação, a equação acima dá a diferença da intensidade total de $\mathrm{P} \& \mathrm{D}$ de um país em relação à média das intensidades, $\bar{I}_{t}$.

Assim, torna-se possível expressar o quanto desta diferença seria atribuída à estrutura industrial do país (a primeira expressão), bem como às intensidades de $\mathrm{P} \& \mathrm{D}$ dentro dos diferentes grupos. 


\subsection{A decomposição para o caso brasileiro}

Pela equação (4) demonstrou-se que a intensidade tecnológica total da indústria resulta da somatória das intensidades de P\&D de cada grupo, ponderadas pela participação do valor agregado destes, $i$, em relação ao valor agregado total da indústria. Os cálculos deste indicador para o Brasil encontram-se na Tabela 1, na qual se analisam os valores para os anos de 2000, 2005 e $2011 .{ }^{10}$

O peso de cada grupo é dado por $w_{i} ; I_{i}$ é a intensidade de $\mathrm{P} \& \mathrm{D}$ em relação ao VTI e o produto desses dois é a contribuição de cada grupo tecnológico para a intensidade total de $\mathrm{P} \& \mathrm{D}$ da indústria. As mudanças na estrutura industrial e/ou variações nos esforços tecnológicos de cada grupo explicam o comportamento da intensidade total.

Ao longo dos anos, a intensidade total da indústria de transformação caiu 0,21 pp., de 2005 em relação a 2000 (1,58-1,79), e recuperou-se no período seguinte, 2011 , em 0,56 pp. $(2,14-1,58)$. Importa saber nesta evolução qual foi o comportamento de cada grupo tanto em termos de suas respectivas participações na estrutura industrial quanto em termos de esforços tecnológicos ( $\mathrm{P} \& \mathrm{D} / \mathrm{VTI})$. Nas últimas três colunas constam as variações de cada componente de análise em relação ao período anterior e são úteis para responder à questão levantada, conforme se demonstrará a seguir.

A queda da intensidade total ocorrida no primeiro período refletiu, em maior proporção, a perda de participação do grupo de alta tecnologia. Pois reduziu-se $2,48 \%$ pp. (8,99\% em 2000 , para $6,51 \%$, em 2005$)$, conforme se vê na coluna "variação do peso relativo dos grupos". Simultaneamente, caiu sua intensidade de $\mathrm{P} \& \mathrm{D}$ em 0,41 pp. (4,46\% em 2000, para 4,05\% em 2005), o que levou à redução da contribuição desse grupo para a intensidade total da indústria (-0,14 pp.). Já o comportamento do grupo de média-alta foi mais estável, com pequenas elevações tanto no peso relativo quanto nos gastos em P\&D em relação ao VTI, o que fez com que sua contribuição para o total da indústria apresentasse pouca variação, de $0,82 \%$ para $0,83 \%$. O grupo de média-baixa tecnologia apresentou substancial

$10 \mathrm{O}$ intuito de se escolher esses anos foi o de acompanhar o comportamento ao longo de toda a década. Ressalta-se que, devido à indisponibilidade de dados anuais da pesquisa, optou-se pelo ano de 2011 por estar mais próximo ao último ano, de 2010, apesar de conter dados do início da década seguinte. 
elevação de seu peso $\left(\mathfrak{W}_{i}\right)$ no total do VTI da indústria de transformação, que variou de $30,06 \%$ para $34,13 \%$.

O comportamento descrito resulta, segundo alguns autores, de um maior dinamismo oriundo do aumento de produção de produtos derivados do petróleo, (Feijó; Lamônica, 2013). Entretanto, observa-se que este relevante aumento não foi acompanhado por maiores gastos em $\mathrm{P} \& \mathrm{D}$, que se reduziram de $1,15 \%$ para $1,01(-0,14 \mathrm{pp})$, fazendo com que a contribuição do grupo para o total da indústria se mantivesse praticamente estável.

Já o grupo de baixa tecnologia, à semelhança do de alta, apresentou queda nos dois componentes: participação relativa (-1,61 pp.) e intensidade de $\mathrm{P} \& \mathrm{D}(-0,23$ pp.), reduzindo a contribuição de $0,23 \%$ para $0,14 \%$ (-0,09 pp.). O ganho de participação do grupo de média-baixa, aliado à perda de peso, sobretudo do grupo de alta, são os fatores mais importantes que explicam a queda da intensidade de P\&D da indústria entre 2000 e 2005. Portanto, a estrutura industrial movimentou-se em direção à maior participação de setores menos intensivos em $\mathrm{P} \& \mathrm{D}$.

Na coluna que mostra a intensidade de $\operatorname{P} \& \mathrm{D}\left(I_{i}\right)$ da Tabela 1 também é possível notar que, entre 2005 e 2011, houve uma elevação do esforço total na indústria de transformação que atingiu 2,14\%, o maior nível para os três períodos. A maior parte desse aumento encontra explicações nos comportamentos dos grupos de média-alta e média-baixa tecnologia. A participação relativa do primeiro aumentou de $26,9 \%$ para $31,56 \%(4,66$ pp.), enquanto que o segundo elevou a intensidade de $\mathrm{P} \& \mathrm{D}$ de $1,01 \%$ para $1,74 \%$ (+0,73 pp.), demonstrando, assim, importante contribuição para a formação da intensidade total. Os outros dois grupos, alta e baixa tecnologia, também elevaram seus esforços tecnológicos associados às perdas de participações no VTI total.

Em particular, no caso do grupo de alta tecnologia, seu peso relativo caiu para 5,11 e isso denota um fraco desempenho, haja vista que no período em análise este grupo apresentou queda contínua.

Enfim, de 2000 para 2011, a elevação da intensidade total deveu-se tanto à maior participação do grupo de média-alta quanto aos maiores esforços do de média-baixa. Em 2005, a variação negativa associa-se ao fraco desempenho do grupo de alta, que perdeu peso em relação ao VTI total; e o ganho de 2011 em relação a 2005 decorreu da maior participação do grupo de média-alta e da maior parcela de gastos em P\&D do de média-baixa. Esses dois grupos contribuíram com $1,66 \%$ dos $2,14 \%$ de intensidade total. 


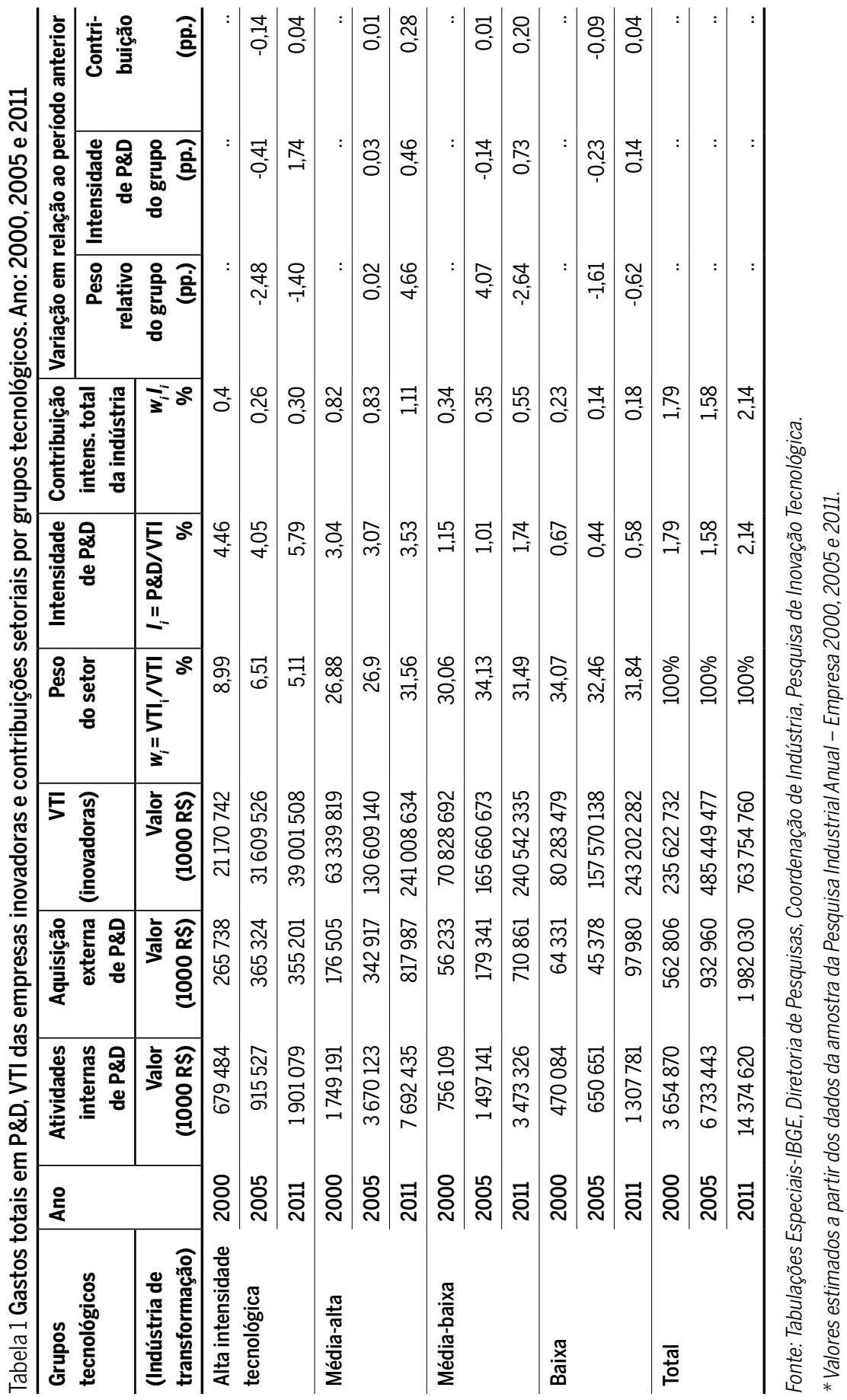


Observa-se que a intensidade total deriva da ponderação das intensidades de cada grupo pelas respectivas participações relativas. Assim, as menores contribuições vêm tanto de participações relativasquantode intensidades de $\mathrm{P} \& \mathrm{D}$ mais baixas. Isso é visto na coluna de contribuição e chama a atenção sobre possíveis impactos potenciais das políticas industriais e de inovação. Alguns exemplos a seguir podem ser observados na tabela e são úteis para corroborar esta última afirmação.

A menor contribuição para a intensidade total de $\mathrm{P} \& \mathrm{D}$ da indústria, vinda do grupo de baixa tecnologia, seria explicada pela sua menor intensidade de P\&D que, por natureza, é uma característica dos setores aí pertencentes.

Da mesma forma, o grupo de alta tecnologia, apesar de ter indicadores de intensidade tecnológica mais elevados, exibe participações relativamente modestas sobre o total do valor adicionado total. A tendência de perda de participação leva à redução da sua contribuição para o total da indústria que, conforme visto acima, apontou decréscimos ao longo do período.

Já o grupo de média-alta tecnologia é o que mais contribui para a formação média da intensidade de $\mathrm{P} \& \mathrm{D}$ da indústria e isso é uma decorrência tanto de sua expressiva participação relativa quanto da intensidade tecnológica. Quanto às opções de políticas, tal exemplo sugere que para um mesmo aumento de esforços tecnológicos a contribuição gerada por tal setor produziria um maior impacto sobre a intensidade total do que em qualquer outro setor. Isso equivale a dizer que o volume de esforços para se alcançar os mesmos níveis de intensidade de $\mathrm{P} \& \mathrm{D}$ agregada é menor para o grupo de média-alta se comparado ao volume necessário para os outros grupos.

Tal condição decorre tanto de sua importância na matriz industrial quanto de suas intensidades tecnológicas. Ao mesmo tempo, os impactos sobre a intensidade agregada poderiam ser produzidos por meio de menores elevações nas participações do setor de média-alta em comparação aos setores de média-baixa e baixa. Isso porque aquele grupo exibe uma combinação expressiva tanto das participações relativas quanto das intensidades de $\mathrm{P} \& \mathrm{D}$.

Essa simplificação não encerra todas as alternativas em termos de políticas para se reduzir as diferenças do Brasil em relação aos países mais adiantados. Pois é importante ressaltar que há também efeitos mútuos entre a estrutura industrial e as intensidades de cada grupo tecnológico cuja importância não deve ser desprezada no desenho e elaboração de políticas. 


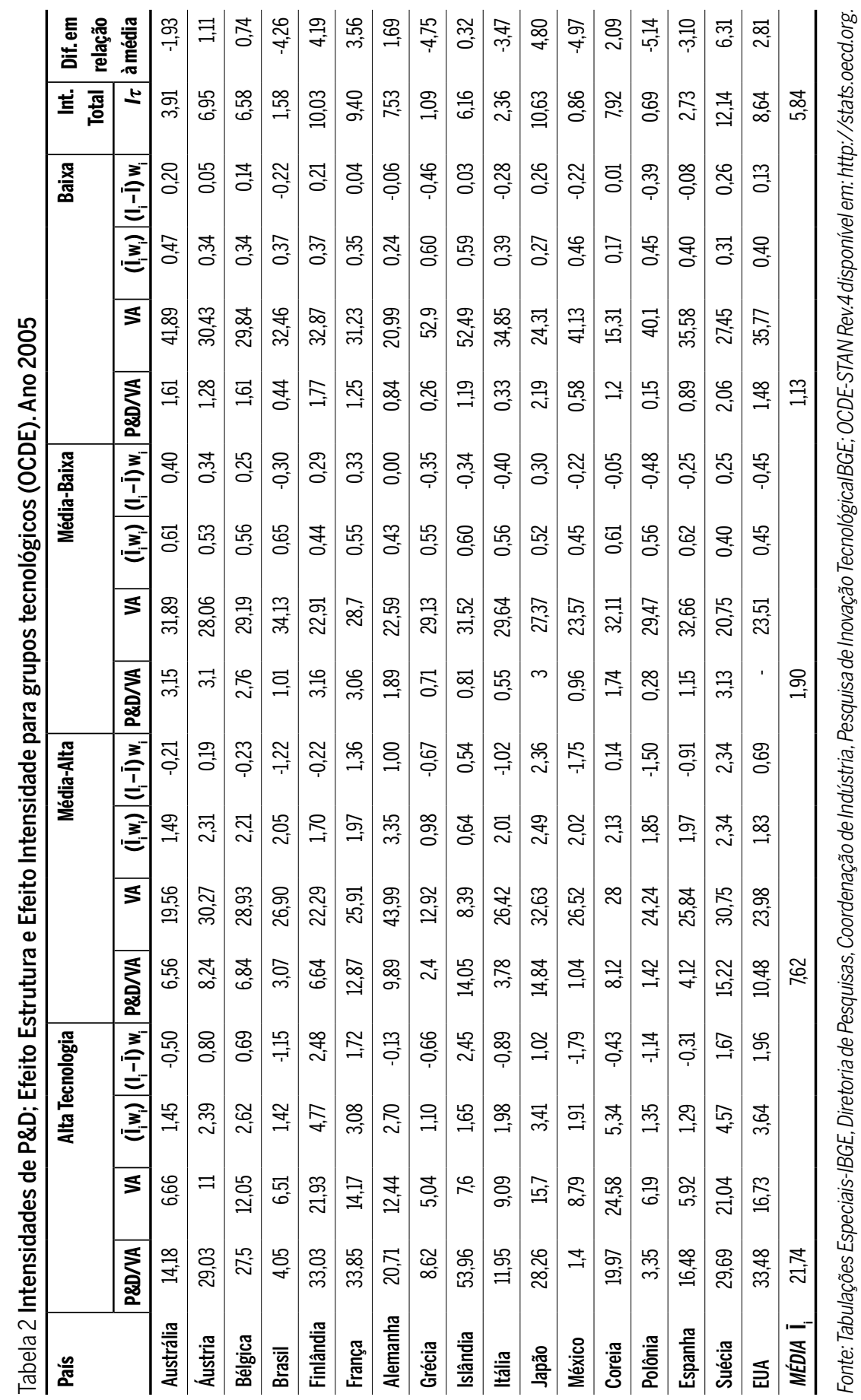




\section{Análise comparativa entre Brasil e outros países}

Os resultados apresentados anteriormente permitem comparações para verificar em que medida a posição do Brasil em relação a outros países, aproximação e/ou afastamento, deve-se à estrutura industrial e à intensidade de P\&D observada em cada grupo tecnológico.

$\mathrm{Na}$ Tabela 2, apresentam-se os cálculos dos componentes de estrutura e de intensidade em cada grupo tecnológico nos países selecionados e os respectivos indicadores de intensidades de $\mathrm{P} \& \mathrm{D}$ para o ano de $2005 .{ }^{11}$

Entre todos os países da amostra, a média das intensidades de P\&D foi de 5,84\% (última linha) e, de acordo com (7) e (8), os cálculos aplicados para o caso do Brasil resultaram nas expressões:

$$
\begin{aligned}
& \sum_{i}^{n}\left[\left(\bar{I}_{i} w_{i}\right)-\bar{I}_{t}\right]=4,48 \%-5,84 \%=-1,36 \% \\
& \sum_{i}^{n}\left[\left(I_{i}-\bar{I}_{i}\right) w_{i}\right]=-2,90 \%
\end{aligned}
$$

O que significa que a diferença da intensidade de P\&D para o Brasil em relação à média é de $-4,26 \%(1,58 \%$ - 5,84\%).

As intensidades de $\mathrm{P} \& \mathrm{D}$ dos grupos tecnológicos respondem por aproximadamente $2,90 \%$ da diferença inferior à média, enquanto que a estrutura industrial responde por aproximadamente 1,36\%. A Tabela 3, mostra os valores para o Brasil.

Uma vez que cada grupo tecnológico é composto por seus respectivos setores, pode-se afirmar que este resultado decorre tanto da forma como se distribui o valor adicionado de cada setor sobre toda a indústria como também de suas intensidades de P\&D. Para melhor compreensão, é possível analisar as participações relativas de alguns setores da indústria em termos de valor adicionado e as respectivas intensidades de P\&D a partir dos dados da tabela.4

Pode ser observado que alguns países que predominam participações mais elevadas em setores mais intensivos em P\&D exibem valores de esforços tecnológicos mais elevados na indústria. A combinação de pesos ex11 Utilizou-se o banco de dados STAN (Structural Analysis Database International Standard Industry Classification, rev. 3) da OCDE para todos os grupos tecnológicos e todos os países da OCDE. Para efetuar os cálculos da intensidade média de P\&D entre os países, a análise teve que se restringir ao último ano para o qual havia disponibilidade de dados referentes aos quatro grupamentos tecnológicos. Sendo assim, o ano de 2005 foi o que se enquadrou nas exigências. 
pressivos em setores de alta e média tecnologia, que, por natureza, efetuam níveis de gastos em $\mathrm{P} \& \mathrm{D}$ mais elevados, contribui para que os esforços agregados da indústria também sejam elevados. Este é o caso da Coreia, que apresenta alta participação no setor de Eletrônica e Comunicações (grupo de alta tecnologia) e, também, da Alemanha. A estrutura industrial deste último país apresenta participações elevadas tanto no setor de máquinas e equipamentos quanto no setor automobilístico,ambos do grupo de média-alta intensidade tecnológica, e não há fortes destaques para o setor de alta tecnologia. Essa combinação eleva a intensidade média entre todos os países.

Tabela 3 Efeito-estrutura, Efeito-intensidade para o Brasil a partir da intensidade média de países selecionados. Ano 2005

\begin{tabular}{|c|c|c|c|c|c|c|}
\hline & $\begin{array}{r}\text { Intensidade } \\
\text { média dos } \\
\text { grupos tec. } \\
\text { Países sele- } \\
\text { cionados }\end{array}$ & $\begin{array}{r}\text { Intensida- } \\
\text { de de P\&D- } \\
\text { Brasil }\end{array}$ & $\begin{array}{r}\begin{array}{r}\text { Peso do } \\
\text { setor }\end{array} \\
\text { Brasil }\end{array}$ & $\begin{array}{r}\text { Efeito da } \\
\text { estrutura } \\
\text { industrial } \\
\text { Brasil }\end{array}$ & $\begin{array}{r}\text { Efeito das } \\
\text { intensi- } \\
\text { dades de } \\
\text { P\&D } \\
\text { Brasil }\end{array}$ & Brasil \\
\hline & $\bar{I}_{i}$ & $I_{t}$ & $w_{i}$ & $\begin{array}{r}\left(\bar{l}_{i} w_{i}\right) \\
(1)\end{array}$ & $\begin{array}{r}\left(I_{i}-\bar{l}_{i}\right) \\
(2)\end{array}$ & $(1)+(2)$ \\
\hline Alta & $21.74 \%$ & $4.05 \%$ & $6.51 \%$ & $1,42 \%$ & $-1,15$ & $0,26 \%$ \\
\hline Média-Alta & $7.62 \%$ & $3,07 \%$ & $26.90 \%$ & $2,05 \%$ & $-1,22$ & $0,83 \%$ \\
\hline Média-Baixa & $1.90 \%$ & $1,01 \%$ & $34.13 \%$ & $0,65 \%$ & $-0,30$ & $0,35 \%$ \\
\hline Baixa & $1.13 \%$ & $0,44 \%$ & $32.46 \%$ & $0,37 \%$ & $-0,22$ & $0,14 \%$ \\
\hline $\begin{array}{l}\text { Intensidade } \\
\text { Total Média } \mathbf{H}\end{array}$ & $5.84 \%$ & & & $4,48 \%$ & $-2,90 \%$ & 1,58 \\
\hline
\end{tabular}

Fonte:Tabulações Especiais-IBGE, Diretoria de Pesquisas, Coordenação de Indústria, Pesquisa de Inovação TecnológicalBGE-OCDE-STAN Rev.4 Disponível em: http://stats.oecd.org.

Nos dois casos anteriores, tratam-se de estruturas produtivas especializadas em poucos setores intensivose que diferem de casos como o dos EUA, por exemplo, onde se caracteriza uma estrutura muito mais diversificada. Os dados da Tabela 4 permitem observar participações relativas importantes (\% VTI) tanto nos setores mais intensivos quanto nos de menor intensidade.

Essa maior diversificação significa que a existência de poucos setores com valores muito elevados não se verifica em níveis semelhantes aos de uma estrutura especializada. Porém, há participações mais elevadas 
de outros setores intensivos, como química, farmacêutica, informática e instrumentação, assim como daqueles menos intensivos, como papel e celulose, alimentos, bebidas e fumo. Há, portanto, uma menor dispersão dos valores em relação à média da indústria.

Tabela 4 Distribuição do valor adicionado e intensidades de P\&D: Brasil x Países

\section{selecionados}

\begin{tabular}{|c|c|c|c|c|c|c|c|c|c|c|}
\hline \multirow{3}{*}{$\begin{array}{l}\text { Indústria de } \\
\text { Transformação } \\
\text { (setor) }\end{array}$} & \multirow{2}{*}{\multicolumn{2}{|c|}{\begin{tabular}{|l|} 
Brasil \\
2005
\end{tabular}}} & \multirow{2}{*}{\multicolumn{2}{|c|}{$\begin{array}{r}\text { Coreia } \\
2007\end{array}$}} & \multirow{2}{*}{\multicolumn{2}{|c|}{$\begin{array}{r}\text { Alemanha } \\
2007\end{array}$}} & \multicolumn{2}{|c|}{ Espanha } & \\
\hline & & & & & & & & 2007 & & 2006 \\
\hline & $\begin{array}{r}\text { P\&D/ } \\
\text { VTI } \\
\%\end{array}$ & $\begin{array}{r}\text { VTI } \\
\%\end{array}$ & $\begin{array}{r}\text { P\&D/ } \\
\text { VTI } \\
\%\end{array}$ & $\begin{array}{r}\text { VTI } \\
\%\end{array}$ & $\begin{array}{r}\text { P\&D/ } \\
\text { VTI } \\
\%\end{array}$ & $\begin{array}{r}\text { VTI } \\
\%\end{array}$ & $\begin{array}{r}\text { P\&D/ } \\
\text { VTI } \\
\%\end{array}$ & $\begin{array}{r}\text { VTI } \\
\%\end{array}$ & $\begin{array}{r}\text { P\&D/ } \\
\text { VTI } \\
\%\end{array}$ & $\begin{array}{r}\text { VTI } \\
\%\end{array}$ \\
\hline Total & 1,58 & & 8,84 & & 7,36 & & 2,95 & & 9,67 & \\
\hline Alimentos, bebidas e fumo & 0.3 & 16,99 & 2.81 & 5.20 & 0.86 & 7.06 & 1.25 & 13.51 & 1.62 & 9.0 \\
\hline Têxtil e vestuário & 0.5 & 3.99 & 1.42 & 3,06 & 2.33 & 1.65 & 1.99 & 3.47 & 1.61 & 2.26 \\
\hline Papel e celulose & 0.60 & 3.17 & 0.37 & 1.50 & 0.65 & 2.05 & 0.71 & 2.51 & 1.02 & 3.33 \\
\hline Coque e refino & 1.17 & 17.34 & 2.23 & 5.10 & 2.13 & 0.78 & 3.22 & 1.77 & 5.83 & 4.30 \\
\hline Química & 1.82 & 8.13 & 9.19 & 7.00 & 8.74 & 6.99 & 2.94 & 6.37 & 48.26 & 8.44 \\
\hline Farmacêutica & 2.37 & 2.76 & 9.68 & 3.46 & 19.84 & 3.24 & 18.42 & 2.94 & 3.45 & 5.34 \\
\hline Borracha e plásticos & 1.3 & 3.31 & 3.04 & 4.31 & 3.81 & 4.47 & 2.00 & 4.08 & 2.23 & 3.82 \\
\hline Siderurgia & - & 6.30 & 1.86 & 9.29 & 1.68 & 3.53 & 1.09 & 4.89 & 1.16 & 2.30 \\
\hline Produtos de Metal & 0.51 & 3.73 & 1.49 & 4.66 & 1.34 & 9.10 & 0.98 & 11.10 & .. & 7.69 \\
\hline Máquinas e equipamentos & 1.4 & 5.54 & 6.93 & 9.70 & 5.77 & 15.90 & 4.13 & 7.40 & 8.45 & 6.50 \\
\hline Informática & 7.23 & 0.54 & 21.57 & 1.07 & 15.41 & 0.85 & 13.77 & 0.25 & 24.20 & 1.28 \\
\hline Eq. Elétricos & 3.06 & 2.77 & 4.64 & 3.76 & 3.86 & 6.74 & 3.68 & 3.80 & 5.01 & 3.23 \\
\hline Telecomunicações & 6.39 & 1.82 & 25.61 & 17.96 & 20.15 & 2.95 & 18.34 & 0.96 & 30.80 & 4.27 \\
\hline Instrumentação & 3.34 & 1.09 & 9.32 & 1.20 & 13.11 & 4.58 & 8.49 & 1.22 & 32.78 & 2.88 \\
\hline Automobilística & 3.84 & 8.87 & 15.77 & 9.30 & 17.37 & 15.00 & 4.87 & 5.70 & 15.46 & 6.60 \\
\hline Outros eq. Transporte & 10.78 & 1.59 & 3.44 & 5.35 & 19.13 & 2.06 & 10.89 & 2.71 & 25.32 & 5.12 \\
\hline Aeroespacial & .. & .. & .. & .. & 27.88 & 1.26 & 24.90 & 0.82 & 26.45 & 3.89 \\
\hline
\end{tabular}

Fonte: Tabulações Especiais-IBGE, Diretoria de Pesquisas, Coordenação de Indústria, Pesquisa de Inovação Tecnológica IBGE-OCDE-STAN Rev.3 Disponível em: http://stats.oecd.org.

No caso da Espanha predominam os setores menos intensivos e assemelha-se mais ao Brasil, onde há maior importância relativa de setores como alimentos e bebidas, refino de petróleo e, em menor proporção, química e automobilística. 
Observações semelhantes podem ser feitas quanto aos valores de intensidade de $\mathrm{P} \& \mathrm{D}$. O perfil do gasto empresarial em $\mathrm{P} \& \mathrm{D}$ é bastante distinto entre os países e pode ocorrer que mesmo não havendo dominância dos setores de alta tecnologia haja valores elevados dos gastos empresariais. É o caso da Alemanha para os setores de farmacêutica, informática e telecomunicações, por exemplo. $O$ caso norte-americano mostra também elevados percentuais para os setores de química, telecomunicações e instrumentos. ${ }^{12}$

As comparações com o Brasil revelam que existe um afastamento dos países mais avançados também em relação aos gastos em $\mathrm{P} \& \mathrm{D}$. E percebe-se que até mesmo em relação a um país menor, como a Espanha, ainda existe significativa distância em termos de esforços tecnológicos para a maioria dos setores, confirmando a dominância do efeito intensidade, visto anteriormente.

Pela equação 8 foi possível obter uma expressão que define em que grau este afastamento atribui-se à estrutura industrial ou às intensidades setoriais de $\mathrm{P} \& \mathrm{D}$. No conjunto de todos os países em que se aplicou aquela decomposição, foi possível a constatação de algumas características similares, selecionando-se aqueles cujo PIB per capita é inferior à U\$30.000,00, nos quais se inclui o Brasil. ${ }^{13}$

Uma daquelas características é a existência de diferenças negativas da intensidade de $\mathrm{P} \& \mathrm{D}$ em relação à média, implicando que valores negativos do efeito-estrutura, em geral, associam-se a valores negativos, também, dos efeitos das intensidades de $\mathrm{P} \& \mathrm{D}$. E isso leva a supor que valores mais elevados do PIB relacionam-se a valores mais elevados de intensidade tecnológica total na indústria.

A Tabela 5, abaixo, permite identificar alguns desses casos como, por exemplo: Grécia, Polônia, Espanha, México, além do Brasil e Itália. ${ }^{14}$

A decomposição apresentada auxilia a entender a diferença do Brasil em termos de esforços tecnológicos privados quando comparado a outros países.

Tomando-se os exemplos anteriores: EUA, Alemanha e Coreia, para os quais o efeito total é positivo, nota-se que os gastos em $\mathrm{P} \& \mathrm{D}$ superam aproximadamente 4 vezes a intensidade média total de $5,84 \%$.

12 Vale observar que para a Coreia os dados são de 2007; para Alemanha, Espanha e EUA, os dados são de 2006 e, para o Brasil, de 2005.

13 Considerou-se a média do PIB per capita em dólares correntes entre o período de 1999 e 2011. 14 O valor do PIB per capita médio calculado para o período em análise situou-se próximo ao limite de corte: U\$29.212,73. 
Talvez uma possível explicação disso, mas que necessitaria de uma análise mais aprofundada e de outros dados,seria que os esforços tecnológicos geralmente estão associados aos tamanhos das economias. Muito embora haja economias menores direcionadas a setores mais intensivos em tecnologia.

Tabela 5 Efeito estrutura, efeito intensidade. Brasil x Países selecionados 2005

\begin{tabular}{l|r|r|r}
\hline País & $\begin{array}{r}\mathbf{E}_{\mathbf{i}} \mathbf{W}_{\mathbf{i}} \mathbf{)}-\tilde{\mathbf{l}}_{\mathbf{i}} \\
\text { Efeito da estrutura } \\
\text { industrial }\end{array}$ & $\begin{array}{r}\left.\mathbf{(}_{\mathbf{i}}-\mathbf{I}\right) \mathbf{w}_{\mathbf{i}} \\
\text { Efeito das intensidades } \\
\mathbf{d e} \mathbf{P} \mathbf{D}\end{array}$ & Efeito total \\
\hline Austrália & $-0,77$ & 0,64 & $-0,14$ \\
\hline Austria & 0,49 & 2,41 & 2,9 \\
\hline Bélgica & 0,62 & 1,92 & 2,54 \\
\hline Brasil & $-1,36$ & $-2,90$ & $-4,26$ \\
\hline Finlândia & 2,0 & 3,99 & 5,98 \\
\hline França & 0,84 & 4,52 & 5,36 \\
\hline Alemanha & 1,39 & 2,09 & 3,48 \\
\hline Grécia & $-1,39$ & $-1,56$ & $-2,95$ \\
\hline Islândia & $-1,16$ & 3,28 & 2,12 \\
\hline Itália & $-0,08$ & $-1,63$ & $-2,95$ \\
\hline Japão & 1,43 & 5,16 & 6,59 \\
\hline México & $-0,15$ & $-3,06$ & $-3,21$ \\
\hline Coreia & 2,78 & 1,1 & 3,88 \\
\hline Polônia & $-0,64$ & $-2,72$ & $-3,35$ \\
\hline Espanha & $-0,59$ & $-0,72$ & $-1,31$ \\
\hline Suécia & 2,25 & 5,85 & 8,1 \\
\hline EUA & 1,17 & 3,43 & 4,6 \\
\hline
\end{tabular}

Fonte: Tabulações Especiais-IBGE, Diretoria de Pesquisas, Coordenação de Indústria, Pesquisa de InovaçãoTecnológicalBGE-OCDE-STAN Rev.4. Disponivel em: http://stats.oecd.org.

Dois aspectos são importantes de se notar nessas economias industrializadas: primeiro é que elas tendem a se concentrar nos setores de alta e/ ou de média-alta tecnologia para os quais constituíram, ao longo de suas trajetórias, vantagens comparativas internas (Furtado; Quadros, 2005). E, segundo, mesmo elas não sendo especializadas em segmentos menos intensivos, ainda assim tendem a exibir esforços tecnológicos superiores às menores economias também nesses segmentos. 
Sendo assim, o maior PIB associa-se, em geral, às intensidades mais elevadas para a maior parte dos setores e, também, ao maior peso dos setores mais intensivos. Essas situações resultam em esforços tecnológicos agregados mais elevados. De forma oposta, nas economias menores, o predomínio de setores menos intensivos, ou de níveis de esforços menores em cada setor, leva à esforços tecnológicos agregados menores.

\section{Conclusões}

Uma primeira conclusão que a análise de decomposição permite seria quanto às implicações políticas. Conforme mencionado na seção 2.2 , entre os objetivos de políticas industriais e de inovação estão: alterar a estrutura produtiva em direção aos setores mais intensivos em tecnologia, aumentar a intensidade tecnológica de determinados setores e, adicionalmente, atuar nas duas frentes. Nesse último caso buscando alterar a estrutura industrial e, ao mesmo tempo, elevar a intensidade tecnológica de alguns setores (Araújo; Cavalcante, 2011).

Pelo lado das políticas de inovação, isso traz implicações quanto aos esforços tecnológicos necessários para reduzir a distância em relação aos países avançados. Pois, em setores com baixa contribuição, os níveis exigidos para haver reduções consistentes daquela distância são mais elevados do que naqueles que apresentam contribuição mais elevada.

Para estes últimos significa dizer que, mantidas as mesmas participações e intensidades dos outros setores, menores acréscimos de esforços tecnológicos resultariam em maiores impactos sobre a intensidade agregada. Enquanto que para um setor cuja contribuição fosse menor seriam necessários maiores acréscimos de esforços tecnológicos.

Por outro lado sendo as menores contribuições explicadas, também, pelas participações relativas de cada setor, exigir-se-ia das políticas industriais e tecnológicas elevar, ou atuar, na preservação dessas participações.

Considerando a diversidade de esforços tecnológicos entre os países, é possível outra conclusão: não havendo eficácia das políticas industriais e de inovação, a distância relativa dos países menos desenvolvidos, ou com estruturas industriais pouco intensivas em tecnologias, tende a aumentar. Em razão de seus baixos esforços tecnológicos, a eficácia ocorreria somente se as políticas promovessem o investimento empresarial em $\mathrm{P} \& \mathrm{D}$ em níveis 
superiores àqueles que já eram atingidos na ausência das mesmas. É nesse caso que haveria contribuição para elevar os esforços totais da indústria. ${ }^{15}$

Embora no Brasil tenha ocorrido importante avanço em termos de políticas, parece não ter havido substancial redução da distância relativa em relação aos países mais desenvolvidos.

Outro ponto a ressaltar da relação exposta anteriormente poderia levar a uma interpretação causal simples em que o tamanho da economia seria visto como uma variável de influência tanto sobre as intensidades setoriais como sobre a estrutura industrial do país. Essas duas, por sua vez, exerceriam efeitos sobre a intensidade total de $\mathrm{P} \& \mathrm{D}$ da indústria de transformação.

Porém, é admissível, também, que a relação entre estrutura industrial e intensidade total da indústria de transformação contemple um efeito em direção oposta. Se cada país tem uma determinada capacidade para realizar P\&D, que se traduz em maiores ou menores competências para tal, isso irá se refletir na intensidade de P\&D da indústria. Permitindo afirmar que a estrutura industrial não é totalmente independente da capacidade de realização de $P \& D$ de um país.

Sob tal aspecto, a estrutura industrial é resultado de um processo complexo que compreende fatores econômicos, sociais, culturais, organizacionais etc. E os maiores ou menores esforços tecnológicos exercerão impactos por serem um dos elementos no processo de inovação que cria, transforma e reproduz tal estrutura. Sendo assim, o engajamento mais forte na produção de bens mais intensivos em tecnologia depende dessa maior ou menor habilidade do país em realizar P\&D.

E o fato de uma estrutura industrial ser mais intensiva em P\&D não significa dizer apenas que há um montante maior de recursos aplicados em tais atividades. Espera-se uma influência da capacidade de P\&D do país também sobre os processos de aprendizado ou competências adquiridas, elementos indispensáveis para o aumento da eficiência na realização da P\&D.

Portanto, trata-se da influência da estrutura industrial sobre as habilidades de $\mathrm{P} \& \mathrm{D}$. Isso leva a crer que existe uma relação mútua entre as intensidades dos grupos tecnológicos e a estrutura industrial.

Assim, os resultados da análise anterior não podem ser entendidos como sugestivos de políticas necessariamente alternativas: ou voltadas à 
transformação completa da estrutura industrial ou apoiadas exclusivamente em instrumentos de apoio à inovação ou $\mathrm{P} \& \mathrm{D}$. A questão central é entender os mecanismos pelos quais a inovação afeta a estrutura produtiva. O entendimento, por exemplo, da capacidade de difusão dos setores mais intensivos sobre o conjunto da estrutura produtiva tem importância fundamental, permitindo identificar quem se apropria das vantagens tecnológicas geradas pelos mesmos: empresas, setores ou a estrutura.

Outra questão seria entender em que extensão os ganhos de vantagens tecnológicas são cumulativos e sustentáveis no tempo, tanto em termos de firmas quanto setores e estrutura. E, simultaneamente, qual seria a flexibilidade de resposta das empresas à inovação; se as firmas existentes no mercado ajustar-se-iam a ela ou se haveria entrada de novas firmas.

A estrutura industrial, portanto, seria afetada pela difusão de novas tecnologias requerendo uma maior absorção dos setores usuários; o acúmulo de habilidades derivado dos ganhos tecnológicos refletiria-se na estrutura industrial, e a estrutura seria afetada por um número maior de empresas ou pela entrada de novas empresas.

Portanto, o entendimento de tais mecanismos é fundamental para a elaboração de resultados mais eficazes das políticas de incentivo à inovação e P\&D no Brasil.

\section{Referências}

ARAÚJO, B.;CAVALCANTE, L. Determinantes dos gastos empresariais em pesquisa e desenvolvimento no Brasil: uma proposta de sistematização. Radar-Tecnologia, Produção e Comércio Exterior-Diretoria de Estudos e Políticas Setoriais de Inovação, Regulação e Infraestrutura, 16, p. 9-18, 2011.

CAVALCANTE, L. An Analysis of the Business Enterprise Research and Development Expenditures Composition in Brazil. Revista Brasileira de Inovação, Campinas (SP), p. 433-458, 2013.

COHEN, W.; LEVINTHAL, D. Innovation and Learning: The Two Faces of R\&D. Economic Journal, p. 569-96, 1989.

DE NEGRI, F.;CAVALCANTE, L. Análise dos dados da PINTEC 2011. IPEA-Nota Técnica Diretoria de Estudos e Políticas Setoriais de Inovação, Regulação e Infraestrutura (DISET), 2013

FEIJÓ, C. A.;LAMÔNICA, M. T. Indústria de transformação e crescimento: uma interpretação para o desempenho da economia brasileira nos anos 1990 e 2000. Revista de Economia e Tecnologia, p. 20-40, 2013.

FURTADO, A.;QUADROS, R. Padrões de intensidade tecnológica da indústria brasileira: um estudo comparativo com os países centrais. São Paulo em Perspectiva, v.19, n.1, p. 70-84, 2005. 
GALINDO-RUEDA, F.;VERGER, F. OECD Taxonomy of Economic Activities Based on R\&D Intensity. OECD Science, Technology and Industry Working Papers, n. 04, p. 6-7,2016.

GRILICHES, Z. Issues in Assessing the Contribution of Research and Development to Productivity Growth. In: GRILICHES,Z. R\&D and Productivity: The Econometric Evidence. Chicago: University of Chicago Press, 1998.p. 17-45.

IBGE. Instituto Brasileiro de Geografia e Estatística. Pintec - Tabulações Especiais, 2000.

IBGE. Instituto Brasileiro de Geografia e Estatística. Pintec - Pesquisa de Inovação Tecnológica, 2000.

IBGE. Instituto Brasileiro de Geografia e Estatística. Pintec - Pesquisa de Inovação Tecnológica, 2005.

IBGE. Instituto Brasileiro de Geografia e Estatística. Pintec -Tabulações Especiais, 2005.

IBGE. Instituto Brasileiro de Geografia e Estatística. Pintec - Pesquisa de Inovação Tecnológica, 2011.

IBGE. Instituto Brasileiro de Geografia e Estatística. Pintec -Tabulações Especiais, 2011.

KAYAL, A. $R \& D$ Intensity: An empirical analysis of its relation to the structure of the manufacturing sector in OECD countries. Dhahran, King Fahd University of Petroleum \& Minerals, Kingdom of Saudi Arabia, 2015.

MATHIEU, A.; LA POTTERIE, B. A note on the drivers of R \& D intensity. Working PaperCenter Emile Bernheim, n.08/002, p.-01-12, Bruxelas-Bélgica, 2008.

PACHECO, C. O Estado da Inovação no Brasil: uma avaliação da inovação empresarial brasileira. Mimeografado, 2009.

PAVITT, K.; PATEL, P. Uneven (and Divergent) Technological Accumulation among Advanced Countries: Evidence and a Framework of Explanation. Industrial and Corporate Change, p. 759-787, 1994.

SANDVEN, T.; SMITH, K. Understanding R\&D intensity indicators - Effects of differences in industrial structure and country size. IDEA-Paper Series STEP GROUP - Studies in technology, innovation and economic policy, v. 14, 1998.

SHEEHAN, J; WYCKOFF, A. Targeting R\&D: Economic and Policy Implications of Increasing R\&D Spending. STI working paper, 24 de jul. de 2003.

ZUCOLOTO, G.;TONETO JR., R. Esforço tecnológico da indústria de transformação brasileira. Revista de Economia Contemporânea, 9(2), p. 337-365, 2005.

\section{Sobre o autor}

Paulo César Brigante - paulo.brigante@gmail.com

Universidade Estadual Paulista - UNESP.

\section{Sobre 0 artigo}

Recebido em 17 de maio de 2016. Aprovado em 23 de janeiro de 2017. 